\title{
Investigation on Performance Characteristics of Petrol Engine Using Alternate Fuel
}

\author{
Savita Patil ${ }^{1}$, Rupesh Kumar Malviya ${ }^{2}$, Kuber Dwivedi ${ }^{3}$, \\ ${ }^{1}$ Department of Mechanical Engineering RKDFIST Bhopal (M.P). \\ ${ }^{2}$ Research Scholar Department of Mechanical Engineering RNTU Bhopal (MP). \\ ${ }^{3}$ Department of Mechanical Engineering SCOPE Engg. College Bhopal (M.P.).
}

\begin{abstract}
In the present scenario the S.I. engines being used in automotives by various manufactures are not properly suitable to out climatic condition. As our country is among tropical countries where the variation in the temperature is having very vast range i. e. from $0^{\circ} \mathrm{C}$ to $48^{\circ} \mathrm{C}$ in various regions of the country. Looking in to this vast varying temperature range it is very difficult to say that which temperature is most suited to operating condition of engines and gives us best performance level as for as SFC and brake power is concerned. In my work I have tried to investigate the best option to run the S.I. engine and simultaneously to maintain the emission norms. Petrol reserves are getting exhausted and it is recommended to find the alternate solution to it. In the present work the potential of methanol is being explored to serve as alternate fuel. This work is carried out with the use of petrol and methanol on a three cylinder, four stroke, petrol Maruti 800 engine connected to eddy current type dynamometer for loading was adopted to study engine power, fuel economy, engine exhaust emissions of hydrocarbon, oxides of nitrogen in the exhaust. The performance results that are reported include brake power and specific fuel consumption (SFC) as a function of engine coolant temperature; i.e. $50^{\circ} \mathrm{C}, 60^{\circ} \mathrm{C}$, $70^{\circ} \mathrm{C}$ and $80^{\circ} \mathrm{C}$ with varying engine speed of $1500,2000,2500$, rpm. Today research and development in the field of gasoline engines have to face a double challenge: on the one hand, fuel consumption has to be reduced, while on the other hand, ever more stringent emission standards have to be fulfilled. The development of engines with its complexity of in-cylinder processes requires modern development tools to exploit the full potential in order to reduce fuel consumption. There are many strategies for improving fuel economy and reducing exhaust emission. Hydrocarbon emission $(\mathrm{HC})$ and carbon monoxide $(\mathrm{CO})$. And finally it is concluded that no remarkable difference is recorded. With use of methanol instead of petrol the variation is marginal and can be attributed to the cycle temp and combustion efficiency. Thus methano $l$ is recommended for use as an alternative fuel for petrol engine in coming future.
\end{abstract}

Key words: Petrol engine, methanol, C.I. engine, SFC And engine speed.

\section{Introduction}

The internal combustion engine is the key to the modern society. Without the transportation performed by the millions of vehicles on road and at sea we would not have reached the living standard of today. We have two types of internal combustion engines, the spark ignition, S.I. and the compression ignition, C.I. Both have their merits. The SI engine is a rather simple product and hence has a lower first cost. The problem with the SI engine is the poor part load efficiency due to large losses during gas exchange and low combustion and thermodynamics efficiency. The C.I. engine is much more fuel efficient and hence the natural choice in applications where fuel cost is more important than first cost. The problem with the C.I. engine is the emissions of nitrogen oxides. As the environmental problems caused by vehicle exhaust emissions become more severe, exhaust gas emission regulations and fuel economy standards become more stringent.

The experimental study is carried out on a three cylinders, four stroke, water cooled petrol engine. Maruti 800 is engine connected to eddy current type dynamometer for loading. My objective of this project is to examine engine performance parameter i.e. specific fuel consumption (SFC), brake power (BP) and also measurement of engine exhaust emission i. e. nitrogen oxides, hydrocarbons at coolant temperature of $50^{\circ} \mathrm{C}$, $60^{\circ} \mathrm{C}, 70^{\circ} \mathrm{C}, 80^{\circ} \mathrm{C}$ and at an engine speed of $1500,2000,2500 \mathrm{rpm}$ with respect to engine load $6,9,12 \mathrm{~kg}$ for fuel used Petrol and Methanol. The results are shown by various graphs i.e. between engine coolant temperature and specific fuel consumption, engine coolant temperature and brake power, engine speed and specific fuel consumption, engine speed and brake power, engine load and specific fuel consumption, engine load and brake power, engine coolant temperature and nitrogen oxide, engine coolant temperature and hydrocarbon, engine speed and nitrogen oxide, engine speed and hydrocarbon. 


\section{Methodology}

Experimental Setup :

The setup consists of three cylinder, four stroke, Petrol engine connected to eddy current type dynamometer for loading. It is provided with necessary instruments for combustion pressure and crank-angle measurements. These signals are interfaced to computer through engine indicator for $\mathrm{P} \theta-\mathrm{PV}$ diagrams. Provision is also made for interfacing airflow, fuel flow, temperatures and load measurement. The set up has stand alone panel box consisting of air box, fuel tank, manometer, fuel measuring unit, transmitters for air and fuel flow measurements, process indicator, load indicator and engine indicator. Rotameters are provided for cooling water and calorimeter water flow measurement. The setup enables study of engine performance for brake power, indicated power, frictional power, BMEP, IMEP, brake thermal efficiency, indicated thermal efficiency, Mechanical efficiency, volumetric efficiency, specific fuel consumption, A/F ratio and heat balance. Windows based Engine Performance Analysis software package "Enginesoft" is provided for on line performance evaluation.

\subsection{Specifications:}

Product

Engine

\section{Dynamometer \\ Piezo sensor \\ Crank angle sensor \\ Engine indicator \\ Temperature sensor \\ Temperature \\ Transmitter \\ Load sensor \\ Fuel flow transmitter \\ Air flow transmitter \\ Rotameter \\ Pump \\ Add on card \\ Software \\ Overall dimensions}

:Engine test setup 3 cylinder, 4 stroke, Petrol (Computerized)

:Make Maruti, Model Maruti 800, Type 3 Cylinder, 4 Stroke, Petrol, water cooled, Power 27.6Kw At $5000 \mathrm{rpm}$, Torque $59 \mathrm{NM}$ at 2500rpm, stroke $72 \mathrm{~mm}$,Bore $66.5 \mathrm{~mm}, 796 \mathrm{cc}, \mathrm{CR} 9.2$

:Type eddy current, water cooled, with loading unit

: Range 5000 PSI, with low noise cable

:Resolution 1 Deg, Speed 5500 RPM with TDC pulse.

:Input Piezo sensor, crank angle sensor, No of channels 2, Communication RS232

: Type RTD, PT100 and Thermocouple, Type K

: Type two wire, Input RTD PT100, Range 0- 100 Deg C,

: Output 4-20 mA and Type two wire, InputThermocouple, Range 0-1200

${ }^{0} \mathrm{C}$,Output $4-20 \mathrm{~mA}$

: Load cell, type strain gauge, range $0-50 \mathrm{Kg}$

: DP transmitter, Range 0-500 mm WC

: Pressure transmitter, Range (-) $250 \mathrm{~mm} \mathrm{WC}$

: Engine cooling 100-1000 LPH; Calorimeter 25-250 LPH

: Type Monoblock

: Resolution12 bit, 8/16 input, Mounting PCI slot

: Enginesoft" Engine performance analysis software

: W 2000 x D 2750 x H $1750 \mathrm{~mm}$

\section{Procedure:}

Experiment was conducted on a three cylinder, four stroke, Petrol Maruti 800 engine which is connected to eddy current type dynamometer for loading. The performance results which include Brake Power (B.P.) and Specific Fuel Consumption (SFC) as a function of engine coolant temperature; i.e. $50^{\circ} \mathrm{C}, 60^{\circ} \mathrm{C}, 70^{\circ} \mathrm{C}$ and $80^{\circ} \mathrm{C}$ are reported. The emissions results reported include the concentrations of hydrocarbon, oxides of nitrogen in the exhaust. The test has been conducted to study the effect of engine temperature on SFC and B.P. with varying engine speed i.e. $1500,2000,2500 \mathrm{rpm}$ with the load of $6,9,12 \mathrm{~kg}$.

Engine coolant temperature has been controlled by controlling cooling water flow rate. The cooling water flow rate for engine is measured manually by rotameter. The values of engine performance parameter are directly obtained by "Engine Soft" software.

A test matrix is created to record the engine performance parameter but main focal point was on specific fuel consumption and brake power of the engine at different engine speed 1500, 2000, $2500 \mathrm{rpm}$ with the engine load of $6,9,12 \mathrm{~kg}$ at engine coolant temperature $50^{\circ} \mathrm{C}, 60^{\circ} \mathrm{C}, 70^{\circ} \mathrm{C}$, and $80^{\circ} \mathrm{C}$. These test have been carried out for both petrol and methanol fuel.

\subsection{Observation And Tabulation}

Table No. 3.1 Test Matrix

\begin{tabular}{|c|c|c|c|}
\hline S.No. & Engine Speed (rpm) & Engine Load(kg) & Engine Temp. $\left({ }^{\circ} \mathrm{C}\right)$ \\
\hline 1 & 1500 & $\begin{array}{c}6 \\
9 \\
12\end{array}$ & $50,60,70,80$ \\
\hline 2 & 2000 & $\begin{array}{c}6 \\
9 \\
12\end{array}$ & $50,60,70,80$ \\
\hline 3 & 2500 & $\begin{array}{c}6 \\
9 \\
12\end{array}$ & $50,60,70,80$ \\
\hline
\end{tabular}


3.1. fuel used: - petrol

Table No. 3.2:- SFC, BP, HC AND NO ${ }_{x}$ emission at engine speed 1500rpm and 6 kg engine load

\begin{tabular}{|l|c|c|c|c|c|c|c|}
\hline S.N. & $\begin{array}{c}\text { Engine } \\
\text { Speed (rpm) }\end{array}$ & $\begin{array}{c}\text { Engine } \\
\text { Load (kg) }\end{array}$ & $\begin{array}{c}\text { Engine Temp. } \\
\left({ }^{\circ} \mathrm{C}\right)\end{array}$ & $\begin{array}{l}\text { SFC in } \\
(\mathrm{g} / \mathrm{kwh})\end{array}$ & $\begin{array}{c}\text { B.P. in } \\
\mathrm{KW}\end{array}$ & $\mathrm{HC}$ in ppm & $\begin{array}{c}\mathrm{NO}_{\mathrm{x}} \text { in } \\
\mathrm{ppm}\end{array}$ \\
\hline \multirow{3}{*}{1.} & \multirow{3}{*}{1500} & \multirow{2}{*}{6} & 50 & 940 & 2.03 & 285 & 636 \\
\cline { 4 - 8 } & & & 60 & 810 & 2.18 & 302 & 653 \\
\cline { 4 - 8 } & & 70 & 850 & 2.03 & 297 & 703 \\
\hline
\end{tabular}

Table No. 3.3:- SFC, BP, HC AND NOx emission at engine speed 2000rpm and 6 kg engine load

\begin{tabular}{|c|c|c|c|c|c|c|c|}
\hline S.N. & $\begin{array}{c}\text { Engine } \\
\text { Speed (rpm) }\end{array}$ & $\begin{array}{c}\text { Engine } \\
\text { Load (kg) }\end{array}$ & $\begin{array}{c}\text { Engine Temp. } \\
\left({ }^{\circ} \mathrm{C}\right)\end{array}$ & $\begin{array}{c}\text { SFC in } \\
(\mathrm{g} / \mathrm{kwhr})\end{array}$ & $\begin{array}{c}\text { B.P. in } \\
\text { KW }\end{array}$ & HC in ppm & $\begin{array}{c}\mathrm{NO}_{\mathrm{x}} \text { in } \\
\text { ppm }\end{array}$ \\
\hline \multirow{2}{*}{1.} & \multirow{3}{*}{2000} & \multirow{3}{*}{6} & 50 & 890 & 2.35 & 312 & 542 \\
\cline { 4 - 8 } & & 60 & 700 & 2.72 & 317 & 614 \\
\cline { 4 - 8 } & & 70 & 720 & 2.69 & 328 & 723 \\
\cline { 3 - 8 } & & & 80 & 700 & 2.82 & 332 & 767 \\
\hline
\end{tabular}

Table No. 3.4:- SFC, BP, HC AND NOx emission at engine speed 2500rpm and 6 kg engine load

\begin{tabular}{|c|c|c|c|c|c|c|c|}
\hline S.N. & $\begin{array}{c}\text { Engine } \\
\text { Speed (rpm) }\end{array}$ & $\begin{array}{c}\text { Engine } \\
\text { Load }(\mathrm{kg})\end{array}$ & $\begin{array}{c}\text { Engine Temp. } \\
\left({ }^{\circ} \mathrm{C}\right)\end{array}$ & $\begin{array}{l}\text { SFC in } \\
(\mathrm{g} / \mathrm{kwh})\end{array}$ & $\begin{array}{c}\text { B.P. in } \\
\mathrm{KW}\end{array}$ & $\mathrm{HC}$ in ppm & $\begin{array}{c}\mathrm{NO}_{\mathrm{x}} \text { in } \\
\mathrm{ppm}\end{array}$ \\
\hline \multirow{2}{*}{1.} & \multirow{3}{*}{2500} & \multirow{3}{*}{6} & 50 & 580 & 3.56 & 375 & 502 \\
\cline { 4 - 9 } & & & 60 & 780 & 3.26 & 376 & 497 \\
\cline { 4 - 8 } & & & 70 & 690 & 3.61 & 374 & 702 \\
\hline
\end{tabular}

Table No. 3.5:- SFC, BP, HC AND NO emission at engine speed 1500rpm and 9 kg engine load

\begin{tabular}{|c|c|c|c|c|c|c|c|}
\hline S.N. & $\begin{array}{c}\text { Engine } \\
\text { Speed (rpm) }\end{array}$ & $\begin{array}{c}\text { Engine } \\
\text { Load (kg) }\end{array}$ & $\begin{array}{c}\text { Engine Temp. } \\
\left({ }^{\circ} \mathrm{C}\right)\end{array}$ & $\begin{array}{c}\text { SFC in } \\
(\mathrm{g} / \mathrm{kwhr})\end{array}$ & $\begin{array}{c}\text { B.P. in } \\
\mathrm{KW}\end{array}$ & $\mathrm{HC}$ in ppm & $\begin{array}{c}\mathrm{NO}_{\mathrm{x}} \text { in } \\
\mathrm{ppm}\end{array}$ \\
\hline \multirow{2}{*}{1.} & \multirow{3}{*}{1500} & \multirow{3}{*}{9} & 50 & 721 & 3.29 & 391 & 898 \\
\cline { 4 - 8 } & & 60 & 640 & 3.19 & 378 & 1210 \\
\cline { 4 - 8 } & & 70 & 644 & 3.01 & 381 & 1267 \\
\cline { 3 - 8 } & & 80 & 624 & 3.11 & 385 & 974 \\
\hline
\end{tabular}

Table No. 3.6:- SFC, BP, HC AND NO emission at engine speed 2000rpm and 9 kg engine load

\begin{tabular}{|c|c|c|c|c|c|c|c|}
\hline S.N. & $\begin{array}{c}\text { Engine } \\
\text { Speed (rpm) }\end{array}$ & $\begin{array}{c}\text { Engine } \\
\text { Load }(\mathrm{kg})\end{array}$ & $\begin{array}{c}\text { Engine Temp. } \\
\left({ }^{\circ} \mathrm{C}\right)\end{array}$ & $\begin{array}{c}\text { SFC in } \\
(\mathrm{g} / \mathrm{kwhr})\end{array}$ & $\begin{array}{c}\text { B.P. in } \\
\mathrm{KW}\end{array}$ & $\mathrm{HC}$ in ppm & $\begin{array}{c}\mathrm{NO}_{\mathrm{x}} \text { in } \\
\mathrm{ppm}\end{array}$ \\
\hline \multirow{2}{*}{1.} & \multirow{3}{*}{2000} & \multirow{3}{*}{9} & 50 & 641 & 3.88 & 398 & 1052 \\
\cline { 4 - 8 } & & 60 & 656 & 4.07 & 406 & 1350 \\
\cline { 4 - 8 } & & 70 & 643 & 3.9 & 396 & 1318 \\
\cline { 3 - 8 } & & 80 & 570 & 4.12 & 392 & 1460 \\
\hline
\end{tabular}

Table No. 3.7:- SFC, BP, HC AND NO ${ }_{x}$ emission at engine speed 2500rpm and 9 kg engine load

\begin{tabular}{|c|c|c|c|c|c|c|c|}
\hline S.N. & $\begin{array}{c}\text { Engine } \\
\text { Speed (rpm) }\end{array}$ & $\begin{array}{c}\text { Engine } \\
\text { Load (kg) }\end{array}$ & $\begin{array}{c}\text { Engine Temp. } \\
\left({ }^{\circ} \mathrm{C}\right)\end{array}$ & $\begin{array}{l}\text { SFC in } \\
\text { (g/kwhr) }\end{array}$ & $\begin{array}{l}\text { B.P. in } \\
\text { KW }\end{array}$ & $\mathrm{HC}$ in $\mathrm{ppm}$ & $\begin{array}{c}\mathrm{NO}_{\mathrm{x}} \text { in } \\
\mathrm{ppm}\end{array}$ \\
\hline \multirow[t]{4}{*}{1.} & \multirow{4}{*}{2500} & \multirow{4}{*}{9} & 50 & 643 & 3.35 & 369 & 803 \\
\hline & & & 60 & 623 & 5.12 & 402 & 1460 \\
\hline & & & 70 & 628 & 3.13 & 386 & 1310 \\
\hline & & & 80 & 610 & 5.28 & 382 & 1065 \\
\hline
\end{tabular}

Table No. 3.8:- SFC, BP, HC AND NO ${ }_{x}$ emission at engine speed 1500rpm and $12 \mathrm{~kg}$ engine load

\begin{tabular}{|c|c|c|c|c|c|c|c|}
\hline S.N. & $\begin{array}{c}\text { Engine } \\
\text { Speed (rpm) }\end{array}$ & $\begin{array}{c}\text { Engine } \\
\text { Load }(\mathrm{kg})\end{array}$ & $\begin{array}{c}\text { Engine Temp. } \\
\left({ }^{\circ} \mathrm{C}\right)\end{array}$ & $\begin{array}{l}\text { SFC in } \\
(\mathrm{g} / \mathrm{kwh})\end{array}$ & $\begin{array}{c}\text { B.P. in } \\
\mathrm{KW}\end{array}$ & $\mathrm{HC}$ in ppm & $\begin{array}{c}\mathrm{NO}_{\mathrm{x}} \text { in } \\
\mathrm{ppm}\end{array}$ \\
\hline \multirow{2}{*}{1.} & \multirow{3}{*}{12} & 50 & 580 & 2.42 & 312 & 1165 \\
\cline { 4 - 8 } & \multirow{2}{*}{1500} & \multirow{2}{*}{12} & 60 & 590 & 4.14 & 328 & 1310 \\
\cline { 4 - 8 } & & & 70 & 600 & 2.32 & 320 & 1366 \\
\cline { 4 - 8 } & & & 80 & 570 & 3.96 & 284 & 1235 \\
\hline
\end{tabular}


Investigation on Performance Characteristics of Petrol Engine Using Alternate Fuel

Table No. 3.9:- SFC, BP, HC AND NO emission at engine speed 2000rpm and $12 \mathrm{~kg}$ engine load

\begin{tabular}{|c|c|c|c|c|c|c|c|}
\hline S.N. & $\begin{array}{c}\text { Engine } \\
\text { Speed (rpm) }\end{array}$ & $\begin{array}{c}\text { Engine } \\
\text { Load (kg) }\end{array}$ & $\begin{array}{c}\text { Engine Temp. } \\
\left({ }^{\circ} \mathrm{C}\right)\end{array}$ & $\begin{array}{c}\text { SFC in } \\
(\mathrm{g} / \mathrm{kwhr})\end{array}$ & $\begin{array}{c}\text { B.P. in } \\
\mathrm{KW}\end{array}$ & $\mathrm{HC}$ in ppm & $\begin{array}{c}\mathrm{NO}_{\mathrm{x}} \text { in } \\
\mathrm{ppm}\end{array}$ \\
\hline \multirow{2}{*}{1.} & \multirow{3}{*}{2000} & \multirow{3}{*}{12} & 50 & 600 & 5.2 & 403 & 1956 \\
\cline { 4 - 8 } & & & 60 & 580 & 5.27 & 418 & 2017 \\
\cline { 3 - 8 } & & & 70 & 560 & 5.54 & 422 & 2096 \\
\hline
\end{tabular}

Table No. 3.10:- SFC, BP, HC AND NO ${ }_{x}$ emission at engine speed 2500rpm and $12 \mathrm{~kg}$ engine load

\begin{tabular}{|c|c|c|c|c|c|c|c|}
\hline S.N. & $\begin{array}{c}\text { Engine } \\
\text { Speed (rpm) }\end{array}$ & $\begin{array}{c}\text { Engine } \\
\text { Load (kg) }\end{array}$ & $\begin{array}{c}\text { Engine Temp. } \\
\left({ }^{\circ} \mathrm{C}\right)\end{array}$ & $\begin{array}{l}\text { SFC in } \\
(\mathrm{g} / \mathrm{kwh})\end{array}$ & $\begin{array}{c}\text { B.P. in } \\
\mathrm{KW}\end{array}$ & $\mathrm{HC}$ in ppm & $\begin{array}{c}\mathrm{NO}_{\mathrm{x}} \text { in } \\
\mathrm{ppm}\end{array}$ \\
\hline \multirow{2}{*}{1.} & \multirow{3}{*}{2500} & \multirow{3}{*}{12} & 50 & 550 & 6.43 & 408 & 2126 \\
\cline { 4 - 8 } & & 60 & 540 & 6.48 & 409 & 2138 \\
\cline { 4 - 8 } & & 70 & 530 & 6.66 & 404 & 2303 \\
\cline { 4 - 8 } & & 80 & 490 & 6.41 & 405 & 2425 \\
\hline
\end{tabular}

\subsection{Fuel Used: - Methanol}

Table No. 3.11:- SFC, BP, HC AND NOx emission at engine speed 1500rpm and $6 \mathrm{~kg}$ engine load

\begin{tabular}{|c|c|c|c|c|c|c|c|}
\hline S.N. & $\begin{array}{c}\text { Engine Speed } \\
(\mathrm{rpm})\end{array}$ & $\begin{array}{c}\text { Engine } \\
\text { Load }(\mathrm{kg})\end{array}$ & $\begin{array}{c}\text { Engine Temp. } \\
\left({ }^{\circ} \mathrm{C}\right)\end{array}$ & $\begin{array}{c}\text { SFC in } \\
(\mathrm{g} / \mathrm{kwhr})\end{array}$ & $\begin{array}{c}\text { B.P. in } \\
\mathrm{KW}\end{array}$ & $\mathrm{HC}$ in ppm & $\begin{array}{c}\mathrm{NO}_{\mathrm{x}} \text { in } \\
\mathrm{ppm}\end{array}$ \\
\hline \multirow{3}{*}{1.} & \multirow{3}{*}{1500} & \multirow{3}{*}{6} & 50 & 1020 & 1.99 & 300 & 650 \\
\cline { 4 - 8 } & & & 60 & 940 & 2.00 & 310 & 670 \\
\cline { 4 - 8 } & & & 70 & 930 & 1.99 & 315 & 715 \\
\hline
\end{tabular}

Table No. 3.12:-SFC, BP, HC AND NO ${ }_{x}$ emission at engine speed 2000rpm and 6 kg engine load

\begin{tabular}{|c|c|c|c|c|c|c|c|}
\hline S.N. & $\begin{array}{c}\text { Engine } \\
\text { Speed (rpm) }\end{array}$ & $\begin{array}{c}\text { Engine } \\
\text { Load }(\mathrm{kg})\end{array}$ & $\begin{array}{c}\text { Engine Temp. } \\
\left({ }^{\circ} \mathrm{C}\right)\end{array}$ & $\begin{array}{l}\text { SFC in } \\
(\mathrm{g} / \mathrm{kwh})\end{array}$ & $\begin{array}{c}\text { B.P. in } \\
\mathrm{KW}\end{array}$ & $\mathrm{HC}$ in ppm & $\begin{array}{c}\mathrm{NO}_{\mathrm{x}} \text { in } \\
\mathrm{ppm}\end{array}$ \\
\hline \multirow{2}{*}{1.} & \multirow{3}{*}{2000} & \multirow{3}{*}{6} & 50 & 920 & 2.29 & 341 & 558 \\
\cline { 4 - 8 } & & & 60 & 750 & 2.58 & 338 & 632 \\
\cline { 4 - 8 } & & 70 & 770 & 2.40 & 342 & 731 \\
\cline { 4 - 8 } & & 80 & 740 & 2.62 & 354 & 782 \\
\hline
\end{tabular}

Table No. 3.13:- SFC, BP, HC AND NO emission at engine speed 2500rpm and 6 kg engine load

\begin{tabular}{|c|c|c|c|c|c|c|c|}
\hline S.N. & $\begin{array}{c}\text { Engine } \\
\text { Speed (rpm) }\end{array}$ & $\begin{array}{c}\text { Engine } \\
\text { Load }(\mathrm{kg})\end{array}$ & $\begin{array}{c}\text { Engine Temp. } \\
\left({ }^{\circ} \mathrm{C}\right)\end{array}$ & $\begin{array}{l}\text { SFC in } \\
(\mathrm{g} / \mathrm{kwh})\end{array}$ & $\begin{array}{c}\text { B.P. in } \\
\mathrm{KW}\end{array}$ & $\mathrm{HC}$ in ppm & $\begin{array}{c}\mathrm{NO}_{\mathrm{x}} \text { in } \\
\mathrm{ppm}\end{array}$ \\
\hline \multirow{2}{*}{1.} & \multirow{3}{*}{2500} & \multirow{3}{*}{6} & 50 & 610 & 3.28 & 395 & 528 \\
\cline { 4 - 8 } & & & 60 & 810 & 3.05 & 385 & 515 \\
\cline { 4 - 8 } & & & 70 & 720 & 3.47 & 392 & 721 \\
\cline { 4 - 8 } & & & 80 & 690 & 3.41 & 399 & 820 \\
\hline
\end{tabular}

Table No. 3.14:- SFC, BP, HC AND NO emission at engine speed 1500rpm and 9 kg engine load

\begin{tabular}{|c|c|c|c|c|c|c|c|}
\hline S.N. & $\begin{array}{c}\text { Engine } \\
\text { Speed (rpm) }\end{array}$ & $\begin{array}{c}\text { Engine } \\
\text { Load }(\mathrm{kg})\end{array}$ & $\begin{array}{c}\text { Engine Temp. } \\
\left({ }^{\circ} \mathrm{C}\right)\end{array}$ & $\begin{array}{l}\text { SFC in } \\
(\mathrm{g} / \mathrm{kwhr})\end{array}$ & $\begin{array}{c}\text { B.P. in } \\
\mathrm{KW}\end{array}$ & $\mathrm{HC}$ in ppm & $\begin{array}{c}\mathrm{NO}_{\mathrm{x}} \text { in } \\
\mathrm{ppm}\end{array}$ \\
\hline \multirow{2}{*}{1.} & \multirow{3}{*}{1500} & \multirow{3}{*}{9} & 50 & 738 & 2.69 & 421 & 1012 \\
\cline { 4 - 8 } & & & 60 & 658 & 2.83 & 399 & 1225 \\
\cline { 4 - 8 } & & 70 & 652 & 2.89 & 392 & 1300 \\
\cline { 4 - 8 } & & 80 & 640 & 3.00 & 401 & 999 \\
\hline
\end{tabular}

Table No. 3.15:- SFC, BP, HC AND NO ${ }_{x}$ emission at engine speed 2000rpm and 9 kg engine load

\begin{tabular}{|c|c|c|c|c|c|c|c|}
\hline S.N. & $\begin{array}{c}\text { Engine } \\
\text { Speed (rpm) }\end{array}$ & $\begin{array}{c}\text { Engine } \\
\text { Load }(\mathrm{kg})\end{array}$ & $\begin{array}{c}\text { Engine Temp. } \\
\left({ }^{\circ} \mathrm{C}\right)\end{array}$ & $\begin{array}{l}\text { SFC in } \\
(\mathrm{g} / \mathrm{kwh})\end{array}$ & $\begin{array}{c}\text { B.P. in } \\
\mathrm{KW}\end{array}$ & $\mathrm{HC}$ in ppm & $\begin{array}{c}\mathrm{NO}_{\mathrm{x}} \text { in } \\
\mathrm{ppm}\end{array}$ \\
\hline \multirow{2}{*}{1.} & \multirow{3}{*}{2000} & \multirow{3}{*}{9} & 50 & 652 & 3.68 & 411 & 1072 \\
\cline { 4 - 8 } & & & 60 & 671 & 3.87 & 424 & 1381 \\
\cline { 4 - 8 } & & & 70 & 660 & 3.0 & 417 & 1329 \\
\cline { 4 - 8 } & & & 80 & 600 & 4.0 & 409 & 1473 \\
\hline
\end{tabular}




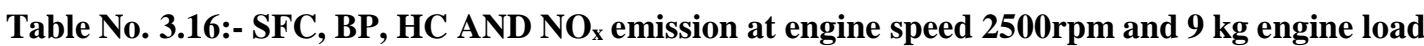

\begin{tabular}{|c|c|c|c|c|c|c|c|}
\hline S.N. & $\begin{array}{c}\text { Engine } \\
\text { Speed (rpm) }\end{array}$ & $\begin{array}{c}\text { Engine } \\
\text { Load }(\mathrm{kg})\end{array}$ & $\begin{array}{c}\text { Engine Temp. } \\
\left({ }^{\circ} \mathrm{C}\right)\end{array}$ & $\begin{array}{l}\text { SFC in } \\
(\mathrm{g} / \mathrm{kwh})\end{array}$ & $\begin{array}{c}\text { B.P. in } \\
\mathrm{KW}\end{array}$ & $\mathrm{HC}$ in ppm & $\begin{array}{c}\mathrm{NO}_{\mathrm{x}} \text { in } \\
\mathrm{ppm}\end{array}$ \\
\hline \multirow{2}{*}{1.} & \multirow{3}{*}{2500} & \multirow{3}{*}{9} & 50 & 652 & 3.20 & 382 & 826 \\
\cline { 4 - 8 } & & & 60 & 642 & 4.89 & 407 & 1485 \\
\cline { 4 - 8 } & & & 70 & 641 & 2.91 & 409 & 1363 \\
\cline { 4 - 8 } & & & 80 & 629 & 4.99 & 403 & 1090 \\
\hline
\end{tabular}

Table No. 3.17:- SFC, BP, HC AND NO emission at engine speed 1500rpm and $12 \mathrm{~kg}$ engine load

\begin{tabular}{|c|c|c|c|c|c|c|c|}
\hline S.N. & $\begin{array}{c}\text { Engine } \\
\text { Speed (rpm) }\end{array}$ & $\begin{array}{c}\text { Engine } \\
\text { Load (kg) }\end{array}$ & $\begin{array}{c}\text { Engine Temp. } \\
\left({ }^{\circ} \mathrm{C}\right)\end{array}$ & $\begin{array}{c}\text { SFC in } \\
(\mathrm{g} / \mathrm{kwhr})\end{array}$ & $\begin{array}{c}\text { B.P. in } \\
\text { KW }\end{array}$ & HC in ppm & $\begin{array}{c}\text { NO }_{\mathrm{x}} \text { in } \\
\mathrm{ppm}\end{array}$ \\
\hline \multirow{3}{*}{1.} & \multirow{3}{*}{1500} & \multirow{3}{*}{12} & 50 & 600 & 2.22 & 328 & 1187 \\
\cline { 4 - 8 } & & 60 & 621 & 4.0 & 341 & 1327 \\
\cline { 3 - 8 } & & 70 & 627 & 2.11 & 331 & 1390 \\
\cline { 3 - 8 } & & 80 & 593 & 3.78 & 300 & 1258 \\
\hline
\end{tabular}

Table No. 3.18:- SFC, BP, HC AND NOx emission at engine speed 2000rpm and $12 \mathrm{~kg}$ engine load

\begin{tabular}{|c|c|c|c|c|c|c|c|}
\hline S.N. & $\begin{array}{c}\text { Engine } \\
\text { Speed (rpm) }\end{array}$ & $\begin{array}{c}\text { Engine } \\
\text { Load (kg) }\end{array}$ & $\begin{array}{c}\text { Engine Temp. } \\
\left({ }^{\circ} \mathrm{C}\right)\end{array}$ & $\begin{array}{l}\text { SFC in } \\
(\mathrm{g} / \mathrm{kwh})\end{array}$ & $\begin{array}{c}\text { B.P. in } \\
\text { KW }\end{array}$ & HC in ppm & $\begin{array}{c}\mathrm{NO}_{\mathrm{x}} \text { in } \\
\mathrm{ppm}\end{array}$ \\
\hline \multirow{2}{*}{1.} & \multirow{3}{*}{2000} & \multirow{3}{*}{12} & 50 & 628 & 5.0 & 398 & 1978 \\
\cline { 4 - 8 } & & 60 & 597 & 5.02 & 399 & 2052 \\
\cline { 4 - 8 } & & 70 & 585 & 5.39 & 404 & 2123 \\
\cline { 4 - 8 } & & 80 & 545 & 5.0 & 418 & 2143 \\
\hline
\end{tabular}

Table No. 3.19:- SFC, BP, HC AND NO emission at engine speed 2500rpm and $12 \mathrm{~kg}$ engine load

\begin{tabular}{|c|c|c|c|c|c|c|c|}
\hline S.N. & $\begin{array}{l}\text { Engine } \\
\text { Speed } \\
(\mathrm{rpm})\end{array}$ & $\begin{array}{c}\text { Engine } \\
\text { Load } \\
(\mathrm{kg})\end{array}$ & $\begin{array}{l}\text { Engine } \\
\text { Temp. } \\
\left({ }^{\circ} \mathrm{C}\right)\end{array}$ & $\begin{array}{l}\text { SFC in } \\
(\mathrm{g} / \mathrm{kw} \\
\mathrm{hr})\end{array}$ & $\begin{array}{l}\text { B.P. } \\
\text { in } \\
\text { KW }\end{array}$ & $\begin{array}{c}\mathrm{HC} \text { in } \\
\mathrm{ppm}\end{array}$ & $\begin{array}{l}\mathrm{NO}_{\mathrm{x}} \\
\text { in } \\
\mathrm{ppm}\end{array}$ \\
\hline \multirow[t]{4}{*}{1.} & \multirow{4}{*}{2500} & \multirow{4}{*}{12} & 50 & 569 & 6.28 & 394 & 2149 \\
\hline & & & 60 & 557 & 6.10 & 389 & 2154 \\
\hline & & & 70 & 541 & 6.32 & 385 & 2321 \\
\hline & & & 80 & 502 & 6.27 & 391 & 2453 \\
\hline
\end{tabular}

\section{Result}

An engine performance characteristic has been determined. The term ,,,performance ${ }^{\text {cee }}$ usually means how well an engine is doing its required task in relation to the input energy or how effectively it provides useful energy in relation to some other comparable engines. It is represented by typical characteristic curves, which are a function of the engine ees operating parameters.

An experiment was conducted on a three cylinder, four stroke, Petrol Maruti 800 engine which is connected to eddy current type dynamometer for loading. The performance results which include Brake Power (B.P.) and Specific Fuel Consumption (SFC) as a function of engine coolant temperature; i.e. $50^{\circ}, 60^{\circ}, 70^{\circ}$, and $80^{\circ} \mathrm{C}$ are reported. The emissions results reported include the concentrations of hydrocarbon, oxides of nitrogen in the exhaust.

Following are the graphs which has obtained for various engine performance parameters:

i. The effect of engine coolant temperature on specific fuel consumption with varying engine speed.

ii. The effect of engine coolant temperature on brake power with varying engine speed.

iii. The effect of engine load on specific fuel consumption with varying engine temperature.

iv. The effect of engine load on brake power with varying engine temperature.

v. The effect of engine coolant temperature on hydrocarbon emission with varying engine speed.

vi. The effect of engine coolant temperature on nitrogen oxide emission with varying engine speed.

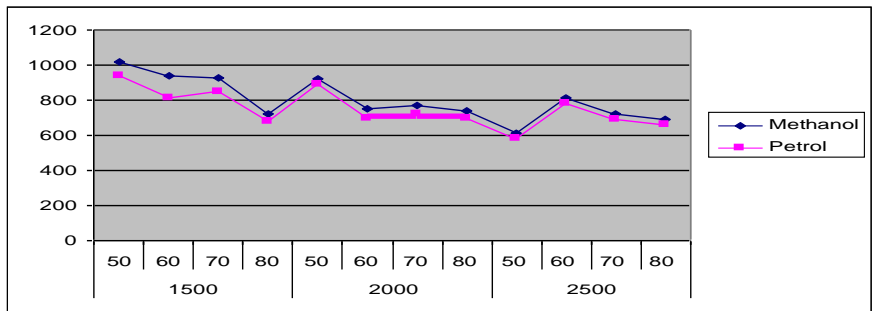


Fig.4.1 The effect of engine coolant temperature on specific fuel consumption with varying engine speed and at 6 kg engine load.

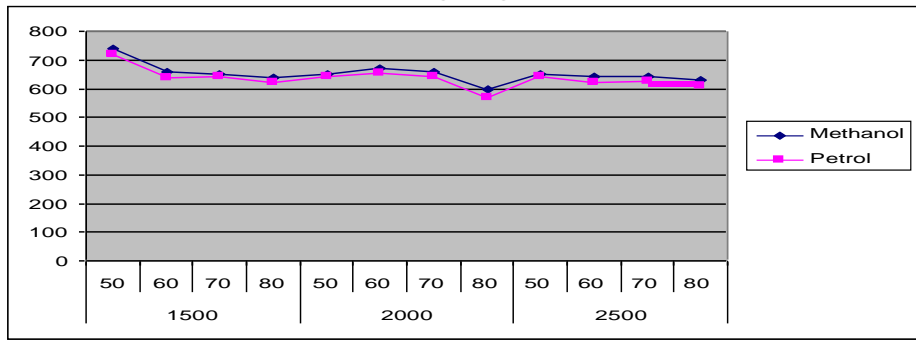

Fig.4.2 The effect of engine coolant temperature on specific fuel consumption with varying engine speed and at $9 \mathrm{~kg}$ engine load.

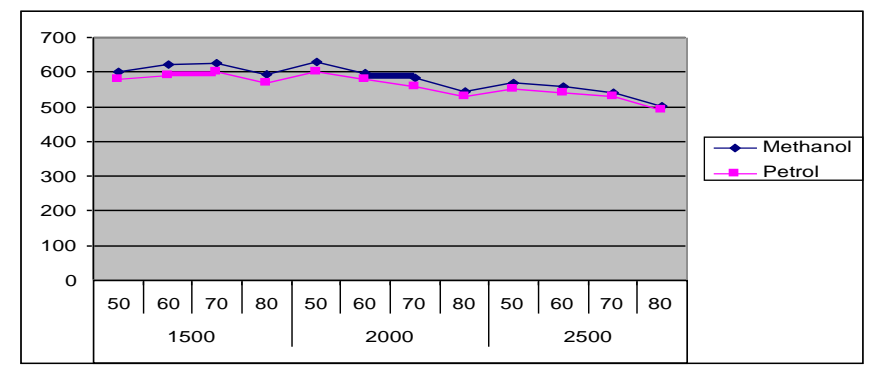

Fig.4.3 The effect of engine coolant temperature on specific fuel consumption with varying engine speed and at $12 \mathrm{~kg}$ engine load.

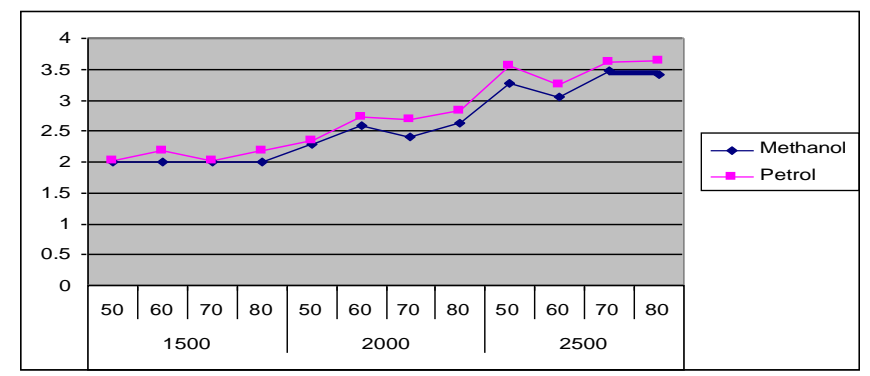

Fig.4.4 The effect of engine coolant temperature on brake power with varying engine speed and at $6 \mathrm{~kg}$ engine load.

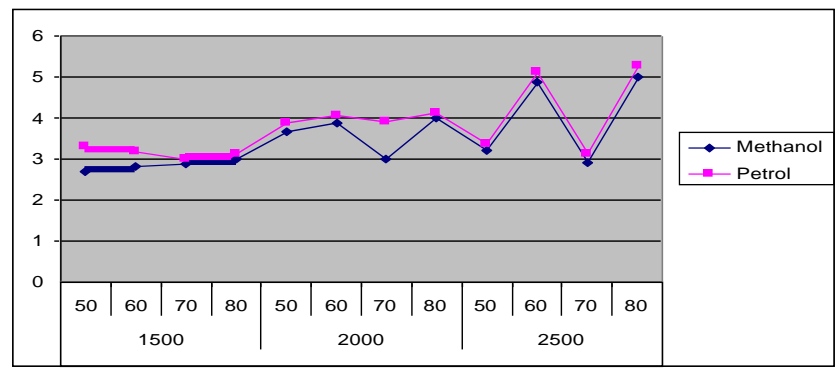

Fig.4.5 The effect of engine coolant temperature on brake power with varying engine speed and at $9 \mathrm{~kg}$ engine load.

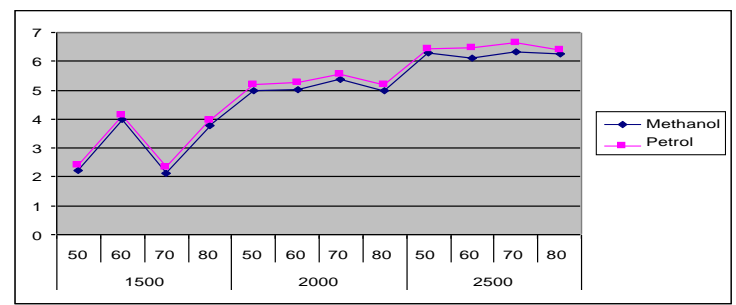

Fig.4.6 The effect of engine coolant temperature on brake power with varying engine speed and at $12 \mathrm{~kg}$ engine load. 
4.2 Effect of engine coolant temperature on SFC and BP at constant speed

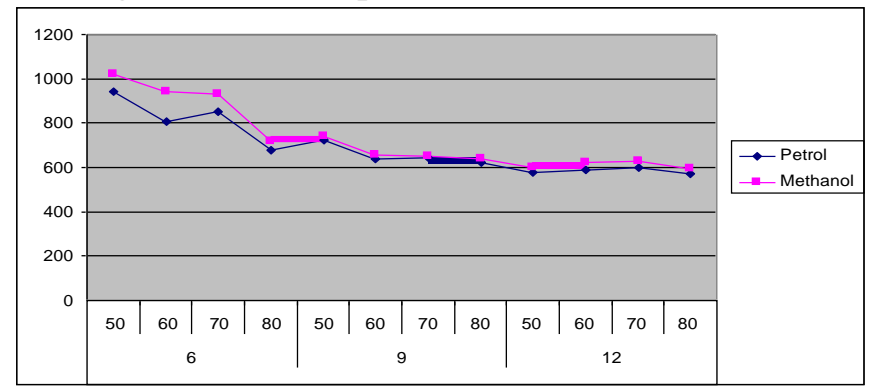

Fig.4.7 The effect of engine load on specific fuel consumption with varying engine coolant temperature and at an engine speed of $1500 \mathrm{rpm}$

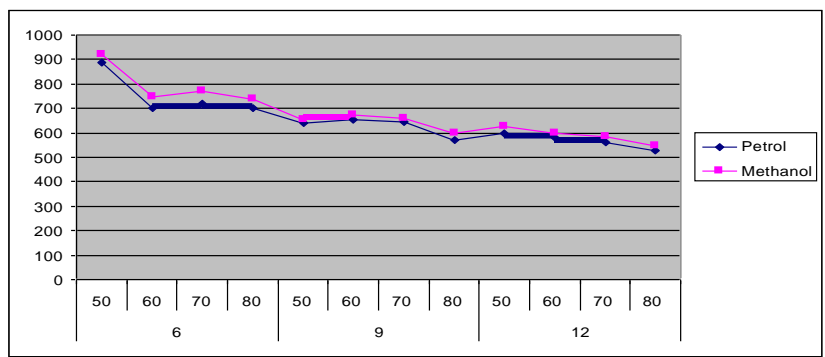

Fig.4.8 The effect of engine load on specific fuel consumption with varying engine coolant temperature and at an engine speed of $2000 \mathrm{rpm}$

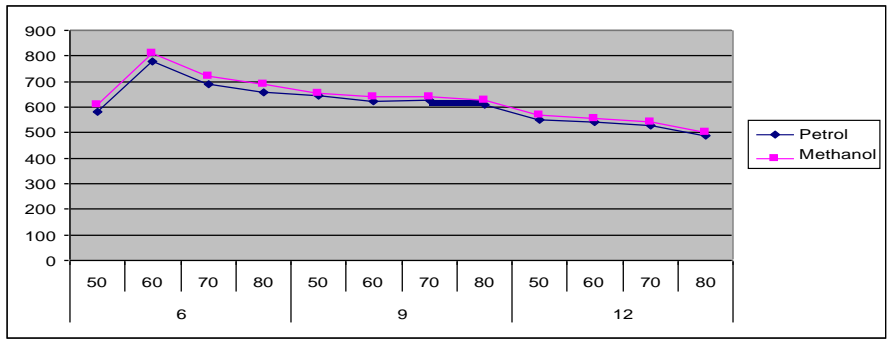

Fig.4.9 The effect of engine load on specific fuel consumption with varying engine coolant temperature and at an engine speed of $2500 \mathrm{rpm}$

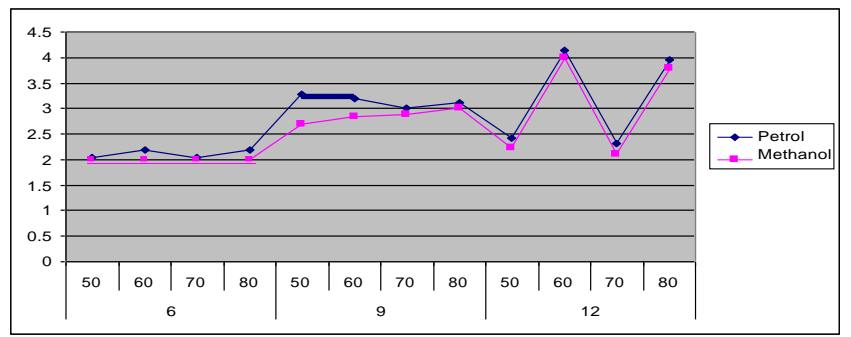

Fig.4.10 The effect of engine load on brake power with varying engine coolant temperature and at an engine speed of $1500 \mathrm{rpm}$

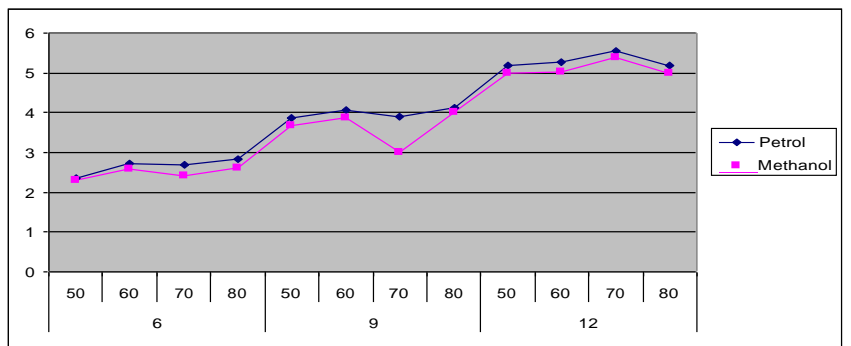

Fig.4.11 The effect of engine load on brake power with varying engine coolant temperature and at an engine speed of $2000 \mathrm{rpm}$ 


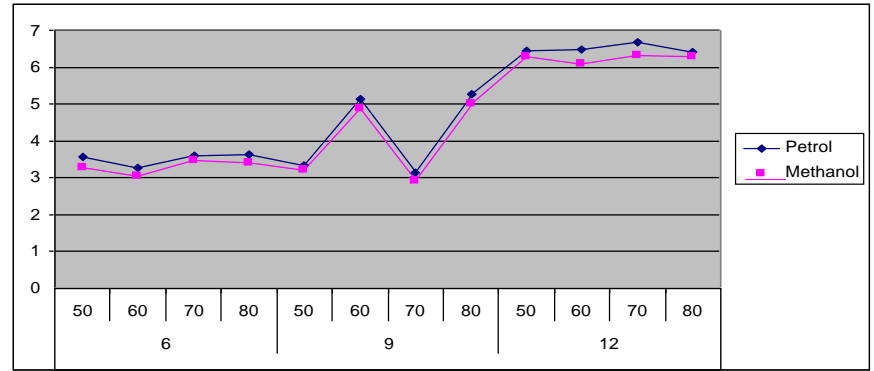

Fig.4.12 The effect of engine load on brake power with varying engine coolant temperature and at an engine speed of $2500 \mathrm{rpm}$

4.3 Effect of engine coolant temperature on $\mathrm{HC}$ and $\mathrm{NO}_{\mathrm{x}}$ at constant load

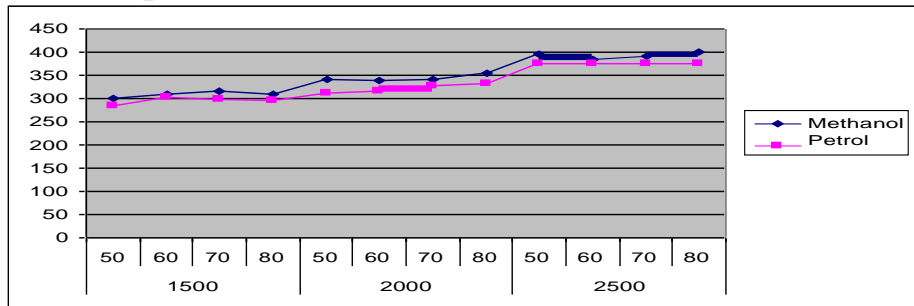

Fig.4.13 The effect of engine coolant temperature on $\mathrm{HC}$ emission with varying engine speed and at an engine load of $6 \mathrm{~kg}$

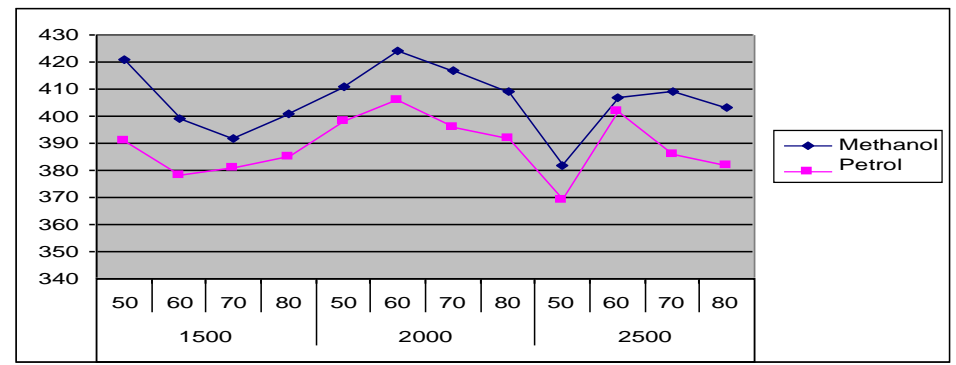

Fig.4.14 The effect of engine coolant temperature on $\mathrm{HC}$ emission with varying engine speed and at an engine load of $9 \mathrm{~kg}$

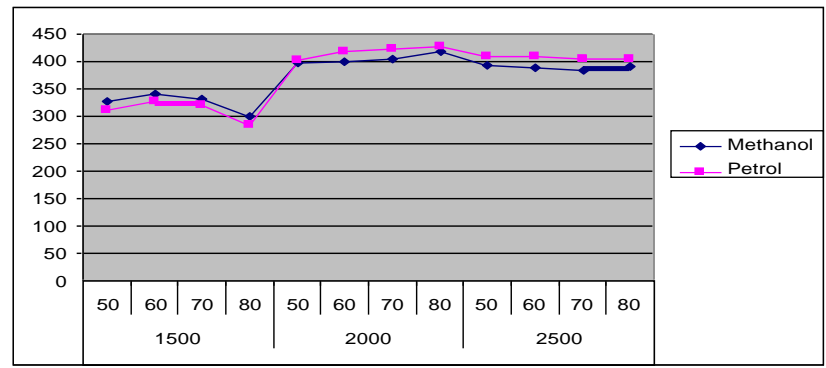

Fig.4.15 The effect of engine coolant temperature on $\mathrm{HC}$ emission with varying engine speed and at an engine load of $12 \mathrm{~kg}$

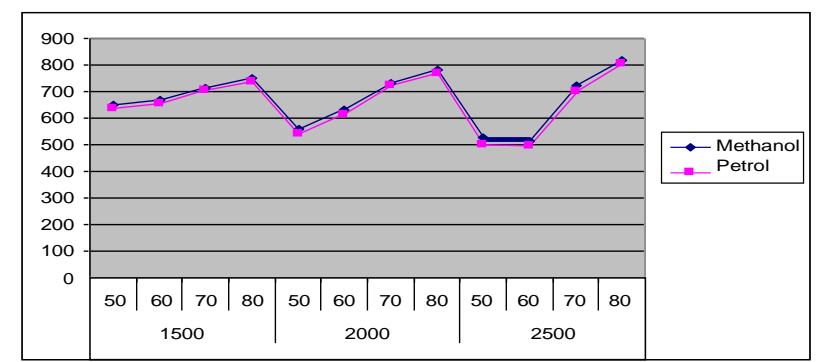

Fig.4.16 The effect of engine coolant temperature on NOx emission with varying engine speed and at an engine load of $6 \mathrm{~kg}$ 


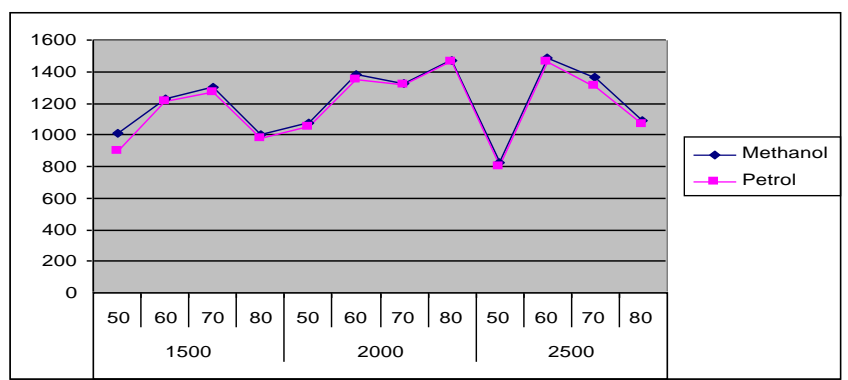

Fig.4.17 The effect of engine coolant temperature on NOx emission with varying engine speed and at an engine load of $9 \mathrm{~kg}$

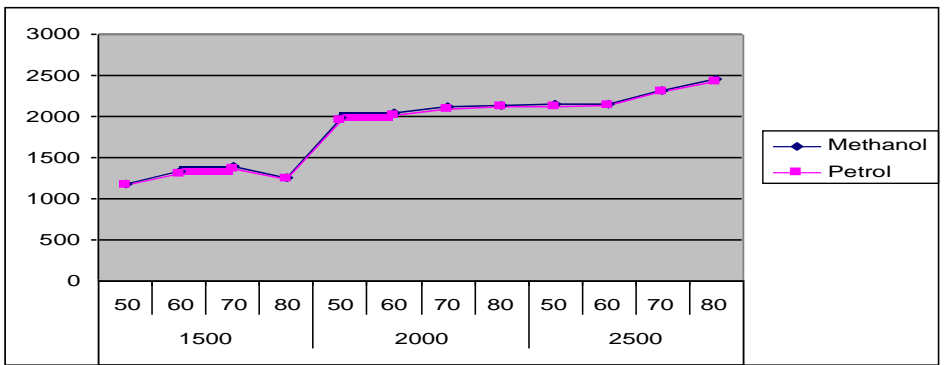

Fig.4.18 The effect of engine coolant temperature on NOx emission with varying engine speed and at an engine load of $12 \mathrm{~kg}$

\section{Conclusion}

An attempt has been made to investigate the potential of methanol as alternate fuel used in petrolengine. A computerised petrol engine test rig of engine test setup with gas analyser has been identified. Experiments have been conducted on three cylinder, four stroke, petrol engine with petrol and Methanol. Readings of specific fuel consumption, brake power, exhaust gas emission has been taken in both cases, i. e. use of petrol and methanol as fuel.

The effect of engine coolant temperature on SFC with varying engine speed is analysed with both fuels (Methanol and petrol). The SFC of methanol is found greater than petrol for all engine coolant temperature range. (i.e. $50^{\circ} \mathrm{C}, 60^{\circ} \mathrm{C}, 70^{\circ} \mathrm{C}, 80^{\circ} \mathrm{C}$ ). However the SFC falls slightly with increase in coolant temperature it is also found that in all cases methanol SFC is more than petrol. For constant load test the maximum variation of SFC occurs in case of 1500 engine rpm, engine coolant temperature $60^{\circ} \mathrm{C}$ and engine load $6 \mathrm{~kg}$. It is observed to be $16.04 \%$.

The effect of engine coolant temperature on BP with varying engine speed is analysed and it is found that for similar conditions for petrol produces more power compare to methanol. It is also observed that with increase in engine speed, BP for both fuels increase for all engine coolant temperature range. For constant load test the maximum variation for BP occurs in case of 2000 engine rpm, engine coolant temperature $70^{\circ} \mathrm{C}$ and engine load $6 \mathrm{~kg}$. It is observed to be $10.78 \%$.

For constant speed test the maximum variation of SFC occurs in case of engine rpm 1500, engine coolant temperature $60^{\circ} \mathrm{C}$ and engine load $6 \mathrm{~kg}$. It is observed to be $8.25 \%$.

For constant speed test, the maximum variation of BP occurs in case of engine rpm 1500, engine coolant temperature $50^{\circ} \mathrm{C}$ and engine load $9 \mathrm{~kg}$. It is observed to be $18.23 \%$.

The effect of engine coolant temperature on $\mathrm{HC}$ emission with varying engine speed for constant load is observed and that is found that the emission of $\mathrm{HC}$ is more for methanol as compared to petrol. For constant load test, the maximum variation of $\mathrm{HC}$ emission occurs in case of engine coolant temperature $50^{\circ} \mathrm{C}$ and engine load $9 \mathrm{~kg}$. It is observed to be $7.67 \%$.

The $\mathrm{NO}_{\mathrm{x}}$ emission for methanol is also observed more as compared to petrol for all speed range. For constant load test the maximum variation of NOx emission occurs in case of engine rpm 1500, engine coolant temperature $50^{\circ} \mathrm{C}$ and engine load $9 \mathrm{~kg}$. It is observed to be $12.69 \%$.

Itis concluded that SFCincreases with methanol fuel and brake power reduces with methanol. The variation is marginal and can be attributed to the cycle temp and combustion efficiency. Thus methanol is recommended for use as an alternativefuel for petrolenginein comingfuture. 


\section{Reference}

[1]. Dr.S.L.Barsley,” Analysis of internal combustion engine heat transfer rate,” IJMET, Vol 3 issue 2(2006), pp. 447-452.

[2]. J.Homstelar,“Heat Transfer in internal combustion Engine," springs, (2008), pp. 963-975.

[3]. Aina T ,Folayan,"influence of engine parameter on performance characterstics of spark ignition engine", Pelagia research (200 8) pp 19151922.

[4]. P.Ravikumar, A.Swarna Kumari," Experimental investigation of IC engine with alternative fuels”, IJAET, 2(1), (2009), pp. 54-58.

[5]. M. M. Kumar Kandasamy, ,'Investigation of the effect of temperature variation on the performance of IC engine ," J.of Sustainable development, (2009) 2(3),pp 176-182

[6]. C.F. Taylor, "The Internal Combustion Engine in Theory and Practice," MIT Press, Cambridge, Massachusetts. (1985)

[7]. Semin, Rosli Abu Bakar,Abdul Rahim Ismail "Investigation of SI engine performance based on simulation," American journal of applied sci., 5(6), (2008),pp. 610-617.

[8]. Descombes,"Study of interaction between mechanical energy and heat transfer applied to IC engine," Applied thermal engineering, (2006),23(16), pp 1320-1327

[9]. Cortona, "Engine thermomanagement for fuel consumption reduction ," Experimental thermal fluid science (2007),30(7),pp 633641

[10]. Alvydas Pikunas, "Influence of engine parameters on performance on spark ignition engine",journal of internal combustion engine,(2003)vol 10(3)

[11]. Reitz Rolf D,“ Advanced internal combustion research ”, Proceedings of the 2000 DOE Hydrogen program review, NREL/CP-570

[12]. Y.R.Suple,"Experimental investigation of exhaust emission of four stroke SI engine," IJMER, 3(5),pp 2600-2605

[13]. H.S. Farkade," Experimental investigation of methanol as alternate fuel for SI engine"IJEATE, 2009, pp 163- 171 